\title{
APLICAÇÃO DOS CONCEITOS KAIZEN/ SMED E TPM PARA REDUÇÃO NO TEMPO DE TESTES EM BANCADAS DE ENGENHARIA DE DURABILIDADE E PERFOMANCE
}

\author{
Vuicik, Rodrigo \\ Wolf, Jairo
}

\section{Diversos tipos de produtos são fabricados diariamente, com linhas robustas e flexíveis, capazes de fabricar por exemplo, centenas de injetores, de inúmeras famílias, para vários clientes.}

\begin{abstract}
The search for productivity is a common objective for companies, in order to reduce production costs. The study proposes to identify production waste, in injection components in engineering test benches to analyze the durability and performance of the products, in order to reduce test times of some components, and consequently the increase in the production capacity of tests, meeting market and company needs. To this end, the present study uses some methods of the KAIZEN and SMED tool, evaluating the current process, proposing improvements and applying it. Despite the current economic crisis, companies are investing in productivity gains. However, the investment capacity decreased, thus generating some limitations in the projects. The results are important and of great value, since there was a substantial improvement in test times and agility in the delivery of technical reports, since at the beginning of the study, the ability to perform tests was analyzed, where cyclic and acyclic activities are considered, concepts were applied and KAIZEN / SMED and TPM methodologies with the objective of standardizing acyclic activities and reducing test waste and applying $R P A$ 's concepts to streamline reporting.
\end{abstract}

\section{RESUMO}

A busca por produtividade é objetivo comum para empresas, a fim de diminuir custos de produção. $\mathrm{O}$ estudo propõe-se em identificar desperdícios de produção, em componentes de injeção em bancadas de testes de engenharia para análise de durabilidade e performance dos produtos, a fim de reduzir tempos de testes de alguns componentes, e consequentemente o aumento de capacidade produtiva de testes, atendendo necessidade de mercado e da empresa. Para tal, o presente estudo, utiliza-se de alguns métodos da ferramenta KAIZEN e SMED, avaliando o processo atual, propondo melhorias $\mathrm{e}$ aplicando. Apesar do momento atual de crise econômica, as empresas investem em ganhos de produtividade. Porém a capacidade de investimento diminuiu, gerando assim algumas limitações nos projetos. Os resultados são importantes e de grande valia, visto que houve melhora substancial nos tempos de testes e agilidade na entrega de relatórios técnicos, pois no início do estudo analisou a capacidade de execução de testes onde considera-se atividades cíclicas e acíclicas, foram aplicados conceitos e metodologias KAIZEN/SMED e TPM com objetivo de padronizar as atividades acíclicas e reduzir os desperdícios nos testes e aplicando conceitos de RPA's para agilizar a emissão de relatórios.

\section{INTRODUÇÃO}

Com o avanço tecnológico presente cada vez mais no mundo industrial, cabe as empresas se adaptarem rapidamente as mudanças, para não perderem credibilidade com seus clientes. Para que isso aconteça, torna-se necessário a utilização de ferramentas de melhoria contínua, com o intuito de deixar a empresa mais competitiva perante o mercado, pois uma corporação que investe em conceitos de melhoria, tende a colher bons frutos no futuro, como redução de custos e aumento de produtividade.

As fábricas, em sua grande maioria, ainda convivem com situações onde há grande dificuldade na resolução de problemas simples. O fato é que mesmo com a chegada de novos conceitos tecnológicos, as empresas ainda dependem de concepções criadas no passado, como por exemplo o 
Sistema Toyota de Produção, adaptado para o então chamado Lean Manufacturing (Manufatura enxuta).

Diante do desafio de tentar reduzir cada vez mais o tempo de testes em bancadas de durabilidade e performance, com o objetivo de realizar mais ensaios para o cliente, torna-se imprescindível a utilização de ferramentas capazes de executar os mesmos sem atrasos e paradas imprevistas, com trocas e setups rápidos, com foco total em melhoraria contínua. Para que esse objetivo seja alcançado, três conceitos devem ser utilizados em conjunto, são eles: o KAIZEN, o TPM e o SMED, ferramentas que estão integradas no conceito Lean.

\section{OBJETIVOS}

- Objetivo Geral:

- Aumentar o número de testes de Durabilidade e Performance no laboratório da engenharia.

- Objetivos Específicos:

- Aumento da produtividade no laboratório;

- Redução de custos com manutenção;

- Setups rápidos em bancadas e motores.

\section{REVISÃO TEÓRICA}

\section{KAIZEN}

A palavra Kaizen, de origem japonesa, tem como significado - "Fazer Bem" (Kai = mudar; Zen = bem). Essa ferramenta ficou amplamente conhecida pela sua utilização dentro do STP (Sistema Toyota de Produção). A ferramenta Kaizen foi criada no Japão pelo engenheiro Taichi Ohno, com a finalidade de minimizar os desperdícios gerados nos processos produtivos, à procura da melhoria contínua, da qualidade dos produtos e o aumento da produtividade (MOREIRA, 2011).

O Kaizen tem como objetivo a melhoria contínua, preza que nenhum dia pode se passar sem que alguma melhoria tenha acontecido seja ela na estrutura da empresa ou no indivíduo. Sua metodologia traz resultados em um curto espaço de tempo e sem grandes investimentos onde conseguimos cada vez mais resultados, apoiados no trabalho e cooperação entre um grupo determinado pela direção da empresa com propósito de alcançar as metas (IMAI, 1994).

Essencialmente, o foco do Kaizen é o modo de pensar de todos os líderes e funcionários, uma atitude de autorreflexão e até mesmo de autocrítica, um cessante anseio de melhorar. É a mudança da situação atual de um processo, sendo analisado e rapidamente implementado, onde as melhorias se traduzem em benefícios concretos (Liker, 2005).

\section{TPM}

O conceito TPM (Manutenção Produtiva Total) teve início no Japão na década de 70, na Nippon Denso KK, mas chegou ao Brasil somente em 1986. Derivada da manutenção preventiva, original dos Estados Unidos, teve sua evolução conforme apresentam NASCIF e KARDEC (2009, p. 193 e 194).

A TPM (Manutenção Produtiva Total) é um método para gerir atividades industriais e foi divulgado principalmente pelos estudos publicados por Seiichi Nakajima, nos anos 1980. Seus objetivos principais são melhorar a eficácia e o tempo de vida útil dos equipamentos, eliminando desperdícios no processo de produção (KOCH, 2007).

Segundo Souza (2007, p. 101), a implantação da metodologia TPM tem como objetivo os seguintes itens:

a) aumentar a eficiência do sistema produtivo, tendo os operadores como agentes de conservação dos seus equipamentos;

b) planejamento, programação e controle das atividades de manutenção corretivas, preventivas e as preditivas;

c) crescimento dos profissionais em função de novos aprendizados, responsabilidades e conscientização;

d) ganhos no gerenciamento da qualidade, confiabilidade e produtividade dos sistemas produtivos;

e) aumentar a eficiência dos profissionais de manutenção e dos departamentos administrativos;

f) gestão da segurança, saúde e meio ambiente.

O TPM é sustentado por 8 pilares, como mostra a figura a seguir:

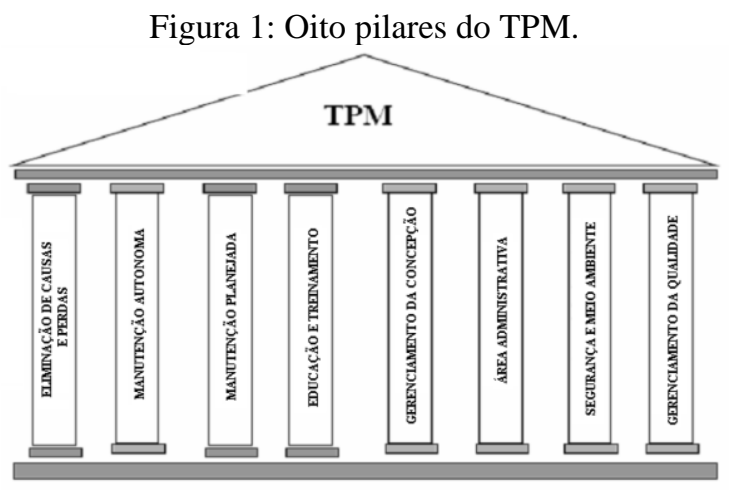

Fonte: Souza (2007).

- Pilar da melhoria especifica: utiliza-se do conceito de manutenção corretiva de melhorias para atuar nas perdas crônicas relacionadas aos equipamentos.

- Pilar manutenção autônoma: baseia-se no treinamento teórico e prático recebidos pelos operários e no espírito de trabalho em equipe para melhoria continua das rotinas de produção e manutenção.

- Pilar manutenção planejada: refere-se às rotinas de manutenção preventiva baseadas no tempo ou condição do equipamento, visando a melhoria contínua da disponibilidade e confiabilidade além da redução dos custos de manutenção. 
- Pilar treinamento e educação: refere-se à aplicação de treinamento técnico e comportamental para liderança, flexibilidade e a autonomia das equipes.

- Pilar eliminar desperdício e identificação de problemas: baseia-se nos conceitos da prevenção da manutenção onde todo o histórico de equipamentos anteriores ou similares é utilizado desde o projeto a fim de se construa equipamentos com índices mais adequados de confiabilidade e manutenção.

- Pilar manutenção autônoma: refere-se à interação da confiabilidade dos equipamentos com a qualidade dos produtos e capacidade de atendimento a demanda. O objetivo deste pilar é também prover aos operadores os conhecimentos e habilidades relativos ao seu equipamento.

- Pilar segurança, saúde e meio ambiente: depende do demais pilares, pois o foco é na melhoria continua das condições de trabalho e na redução dos riscos de segurança e ao meio ambiente.

- Pilar de melhoria nos processos administrativos: utilizando como conceito de organização e eliminação de desperdício nas rotinas administrativas, que de alguma maneira interferem em custos.

\section{SMED}

Segundo MATTANA; PASA (2012), que cita Shingo, deve-se notar que, por razões históricas associadas à evolução da técnica, a TRF também é conhecida por Single Minute Exchange of Die (SMED), ou Troca Rápida de Ferramentas. Shingo apresenta o seu processo de redução de tempo por meio da troca de ferramentas em quatro estágios (MATTANA; PASA, 2012).

De acordo com Sugai, McIntosh e Novaski (2007), a metodologia é considerada a principal referência quando se trata de redução dos tempos de setup de máquinas. Satolo e Calarge (2008) afirmam que o tempo de setup pode ser definido como o tempo transcorrido para realização de todas as tarefas necessárias para a troca de ferramenta, desde o momento em que se tenha completado a última peça do lote anterior, até o momento em que, dentro do coeficiente normal de produtividade, se tenha feita a primeira peça do lote posterior.

As etapas executadas em cada fase de implantação do SMED são: i) estágio preliminar: nesse estágio, não há qualquer distinção entre setups interno e externo, somente é analisado o procedimento atual de setup; ii) estágio um: é o estágio mais importante na implantação do SMED, no qual separam-se as atividades em internas e externas; iii) estágio dois: durante esse estágio, analisam-se as operações de setup para definir se alguma das atividades de setup interno pode ser transformada em setup externo; iv) estágio três: no último estágio de implantação do SMED, são analisadas todas as atividade de setup interno e externo para buscar possíveis oportunidades de melhorias, levando em conta a mitigação de ajustes e a padronização dos métodos de fixação. Ainda segundo Shingo (1996), o SMED conduz a melhoria do setup de forma progressiva. Assim, ele passa pelos quatro estágios básicos, conforme visto na Figura 2 (PIRISOTTO; PACHECO, 2016).

\section{ESTUDO DE CASO}

No atual momento em que o mercado industrial passa, compete às empresas buscar cada vez mais alternativas que visam um melhor atingimento de produtividade. Isso está diretamente ligado no tempo em que o técnico atua ao realizar setups em bancadas, bem como as paradas imprevistas geradas por manutenção das mesmas.

Para reduzir o desperdício de tempo dos técnicos na realização de atividades acíclicas, foram realizados alguns levantamentos de dados para entender melhor a empregabilidade de tempo dos colaboradores desde o momento em que as peças chegam para serem testadas, até a conclusão do relatório. Com isso, pôde-se observar que havia um grande desprendimento de tempo dos técnicos na realização do setup, não só nas bancadas, como também durante a preparação dos produtos a serem ensaiados.

A empresa da qual o estudo propõe redução do tempo de testes, além do aumento da capacidade produtiva, é uma grande indústria do ramo de autopeças, fabricante de bombas e injetores a Diesel, para veículos de estrada e fora de estrada.

Diversos tipos de produtos são fabricados na empresa diariamente, com linhas robustas e flexíveis, capazes de fabricar por exemplo, centenas de injetores, de inúmeras famílias, para vários clientes, numa mesma linha de produção.

\section{Ferramentas de melhoria com foco na produtividade}

Após a fabricação do produto, os mesmos são enviados para o departamento da engenharia, na área de confiabilidade, para que seja testado performance e durabilidade em bancadas de teste, onde os injetores são simulados em condições reais de um veículo. Esses testes são realizados com base em instruções e prescrições acordadas com o cliente.

Normalmente os produtos chegam completos para serem testados, ou seja, da mesma forma como são enviados para os consumidores. Em determinadas situações, podem vir somente alguns componentes desse produto.

É dado início ao setup do teste no momento em que o técnico pega as peças para prepará-las. Durante essa preparação, deve-se desmontar certos componentes antes de montar os injetores na bancada. Com isso, as peças desmontadas tornam-se obsoletas para o teste. Em função disso, foi criada através de uma ferramenta de melhoria contínua, uma ideia que abstrai estes componentes na linha de montagem do produto, somente no caso de serem ensaiadas no laboratório da engenharia. Essa ideia faz com que não só a produção tenha uma maior produtividade na hora de montar os produtos, como também os técnicos no momento de prepará-las na bancada. 
Além dessa, muitas outras ideias são criadas diariamente com uso das ferramentas de melhoria contínua. A empresa investe forte nessas ferramentas, pois é a partir delas que o colaborador pode dar sua sugestão para beneficiar não só a corporação, como os respectivos colaboradores.

\section{Utilização do TPM em bancadas}

Os testes são executados em bancadas que simulam a situação mais crítica do produto. Em alguns casos, certos componentes do produto chegam a fraturar devido ao alto esforço exigido no teste. Desta forma, elementos da máquina sofrem com a carga aplicada e se quebram ou desgastam com o tempo.

Quando ocorre uma quebra na bancada, o técnico responsável abre uma ordem para a manutenção como máquina parada. Em casos de a peça vir através de importação, ocorre de faltar no estoque. Dessa forma, a bancada fica parada no aguardo da chegada da nova peça.

Com o objetivo de amenizar esse desconforto e aumentar a produtividade, foi implementado o TPM em bancadas de durabilidade e performance na engenharia. Assim consegue-se estimar com quanto tempo um elemento de máquina irá fraturar, graças à manutenção preditiva.

Após inserido o conceito nas bancadas, os testes podem ocorrer da forma como foram planejados, evitando perdas por paradas e fugas de rotina. Assim, é possível ter vantagem competitiva, pois não tem um volume considerável de interrupções. Além disso, os colaboradores passam a ter uma mentalidade preventiva, caracterizada pela manutenção autônoma.

\section{Relatórios através de RPA's}

Depois de realizar os testes em bancadas, o técnico inicia a confecção do relatório com dados coletados durante o teste, para que então, possa encaminhar essas informações ao engenheiro responsável por realizar os cálculos que determinam a vida útil de cada componente do produto. Além dos dados técnicos, o engenheiro acrescenta gráficos e tabelas, fotos de desgaste, imagens capturadas através do MEV (Microscópio Eletrônico de Varredura), para identificar possíveis trincas e informações adicionais.

A confecção do relatório demanda um elevado tempo do engenheiro, pois o mesmo tem que inserir tabelas, gráficos, cálculos e fotos manualmente. O tempo empregado na elaboração do relatório é tido como um desaproveitamento, já que o engenheiro poderia investir esse tempo por exemplo, na realização de cálculos, na busca de novas melhorias no produto ou até mesmo no aumento de sua produtividade.

Com o foco em aumentar a produtividade do engenheiro, surgiu então a ideia de implementar o conceito do RPA (Automação de Processos Robóticos) na elaboração dos relatórios. Para que isso aconteça, o técnico precisa inserir por exemplo, todas os dados coletados durante o teste (MEV, fotos, tabelas), nomeadas corretamente, para que o software consiga procurá-las no sistema e ordená-las de forma coerente, no corpo do relatório. Após isso, basta o engenheiro completar o mesmo com as informações que lhe forem cabíveis. Dessa forma, o tempo de atuação na elaboração do relatório reduz e, consequentemente, a produtividade aumenta.

\section{RESULTADOS}

Os resultados obtidos com a utilização das ferramentas Lean foi muito positivo. Observou-se um aumento gradual de produtividade e uma redução em ordens abertas para a manutenção de máquinas.

Se comparar o primeiro trimestre do ano de 2018 e 2019, com o mesmo período do ano de 2020, verificou-se uma redução de cerca de $50 \%$ em ordens abertas para a manutenção, por conta de máquinas paradas. Isso só foi possível graças a implementação do TPM nas bancadas e motores de durabilidade e performance.

O gráfico a seguir ilustra essa redução expressiva no número de ordens abertas para a manutenção:

Gráfico 1 - Comparativo de ordens abertas por máquina parada 2018 a 2020.

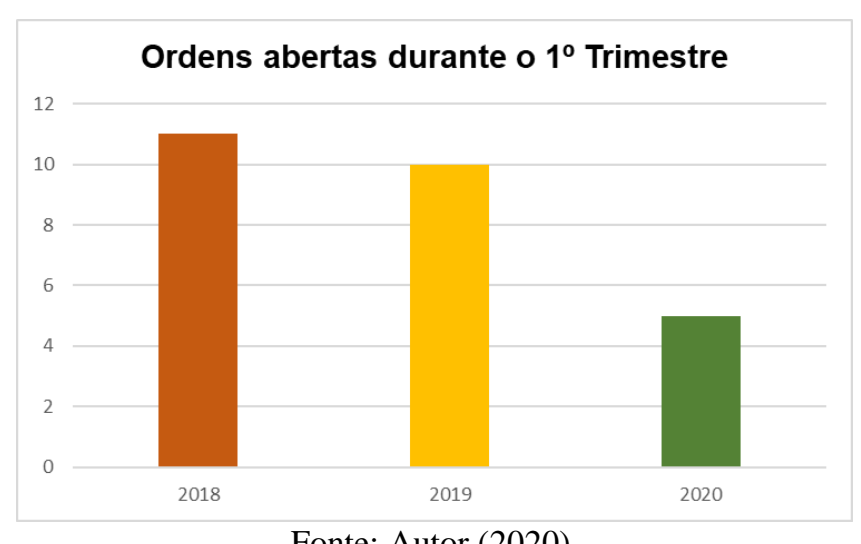

Fonte: Autor (2020).

Além da redução em ordens abertas por máquina parada, houve também uma redução de tempo na confecção de relatórios, do qual utilizou-se o conceito RPA. Antes da implementação, demorava-se em torno de 8 horas para elaborar um relatório manualmente, de acordo com sua complexibilidade. Após a implementação deste conceito, o tempo caiu para cerca de 1 hora, levando em conta um relatório com aproximadamente 170 fotos. Com isso, foi possível obter cerca de $87 \%$ de redução na elaboração do relatório, assim o engenheiro consegue desenvolver outras atividades e aumentar sua capacidade produtiva.

\section{CONCLUSÃO}

Em virtude da utilização das ferramentas Lean Manufacturing, foi possível enxergar a real necessidade que a indústria tem para resolver os problemas encontrados pelo caminho, sejam eles na linha de produção, ou até mesmo na engenharia.

Observa-se que para solucionar estes problemas, basta escolher a ferramenta mais compatível com o tema 
abordado. Neste caso, as ferramentas Kaizen, SMED e TPM foram as escolhidas e conseguiram sanar com êxito as barreiras encontradas. Notou-se que com as respectivas utilizações, cada uma com seu foco específico, foi possível obter reduções de peças, diminuição de ordens abertas para a equipe de manutenção e aumento de produtividade. Todos esses benefícios levam a um único objetivo: reduzir o tempo dos testes de durabilidade e performance. Sem estas ferramentas, não seria possível obter resultados tão convincentes como os vistos.

A melhoria contínua é como um processo de aprendizagem, ela precisa estar intrínseca no planejamento da organização, e deve também, ser levada a sério, por todos que a utilizam. E para que se obtenha sucesso é necessário além da constante motivação dos funcionários, a inserção do programa de melhoria com a cultura organizacional, a fim de obter uma alta produtividade nos processos envolvidos.

\section{REFERÊNCIAS}

IMAI, Masaaki. Kaizen, A estratégia para o sucesso competitivo. São Paulo: Editora Imam, 1994.

KARDEC, A.; NASCIF, J. Manutenção: Função Estratégica, Rio de Janeiro: Qualitymark, 2009.

$\mathrm{KOCH}$, A. Discover the hidden machine: OEE for production team. Amsterdam: FullFact BV, 2007.
Liker, J.K. (2005). O Modelo Toyota, Porto Alegre: Bookman

MATTANA, F. A.; PASA, G. S. Troca rápida de ferramentas: implementação de uma sistemática ampliada. 2012. 25 f. TCC (Graduação) - Curso de Engenharia, Universidade Federal do Rio Grande do Sul, Porto Alegre, 2012. Disponível em: <https://www.lume.ufrgs. br/bitstream/handle/10183/65647/000857909.pdf?sequence =1>. Acesso em: 19/05/2020.

MOREIRA, S. P. S. (2011); Aplicação das ferramentas Lean - Caso de Estudo. Dissertação de Mestrado, Mestrado em Engenharia Mecânica, Instituto Superior de Engenharia de Lisboa, Lisboa, Portugal

PARISOTTO, C.; DE JESUS PACHECO,D.A. Método SMED: Análise e aperfeiçoamento. Dirección y Organización, n. 60, p. 4-23, 2016.

SOUZA, Valdir Cardoso. Organização e gerência da manutenção. 2. ed. Cidade: All Print, 2007.

SUGAI, M.; MCINTOSH, R. C.; NOVASKI, O. Metodologia de Shigeo Shingo (SMED): análise crítica e estudo de caso. Revista Gestão \& Produção, São Carlos, v. 14, n. 2, p. 323-335, maio/ago. 2007. 